\title{
Les effets de la politique sur la santé
}

\section{Yvonne Gilli}

Dre méd., présidente de la FMH

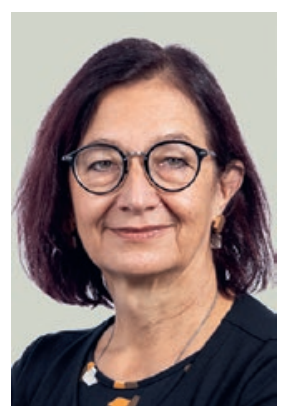

Beaucoup se rendent compte pour la première fois que la politique de santé ne touche pas seulement des thèmes abstraits, discutés «là-bas», à Berne, mais qu'elle a des incidences directes sur la santé. D’elle dépend la possibilité d'avoir accès ou non à un vaccin.

On comprend dès lors que la session de printemps qui s'est achevée le 19 mars ait essentiellement porté sur les questions de politique vaccinale. Les achats de vaccins, en particulier, ont nourri la critique. Malgré tous les retards de livraison et les autorisations en suspens, le Conseil fédéral a réaffirmé l'objectif de vacciner toutes les personnes qui le souhaitent d'ici la fin juin [1]. Il reviendra toutefois à d'autres - cantons et médecins - de tenir cette promesse. Ils ne recevront les lots de vaccins que plus tard, mais devront les administrer malgré tout dans ce délai, et les cabinets médicaux avec une rémunération qui ne couvre pas leurs frais.

Du fait de l'actuelle focalisation sur la pandémie, les autres objets traités au Parlement en lien avec la santé ne suscitent qu'une faible attention, voire aucune. Et parmi ces objets, on trouve le premier volet de mesures visant à maîtriser les coûts. Or, les décisions prises en la matière ont enclenché un processus politique dont les conséquences pourraient nous occuper bien au-delà de la crise actuelle.

Les suites du premier volet de mesures visant à maîtriser les coûts pourraient nous occuper au-delà de la crise du coronavirus.

En toute discrétion, le Parlement a donc décidé qu'il existerait désormais deux structures tarifaires distinctes pour un même ensemble de prestations: une structure pour les prestations individuelles et une autre pour les forfaits. Le potentiel de modération des coûts des forfaits ambulatoires a été bradé inutilement, car une base de coûts distincte pour les tarifs forfaitaires crée de faux incitatifs et finance des structures hospitalières coûteuses là où des cabinets médicaux seraient suffisants. Quant aux efforts en vue d'une structure tarifaire unifiée pour toutes les prestations ambulatoires effectuées en Suissey compris les forfaits - il reste au moins la perspective de pouvoir négocier des forfaits sur une base volontaire.
Il n'a hélas pas été possible, contrairement aux prévisions, d'achever le traitement de l'article autorisant les projets pilotes durant la session de printemps. Les débats intenses n'ont pas permis de décider s'il fallait autoriser exclusivement les projets portant sur les questions de coûts telles que définies dans la loi, ou si des projets visant à promouvoir la numérisation et la qualité pouvaient entrer en ligne de compte. La décision sera vraisemblablement prise cet été.

\section{Un plafond de coûts ne ferait pas que limiter} les prestations médicales, il serait contraire à la Constitution.

Alors que l'on touche au but pour certains dossiers, le travail commence pour l'objet qui, au sein du premier volet de mesures, aura les plus vastes conséquences pour notre système de santé: les «mesures visant à piloter les coûts», par lesquelles le Conseil fédéral voudrait obliger les partenaires tarifaires à définir par avance les coûts «justifiés» pour une année civile, en prévoyant des sanctions financières pour tout dépassement. A ce propos, l'article 47c LAMal a été rejeté de justesse lors de son premier examen en octobre 2020 devant le Conseil national. La Commission de la santé du Conseil des Etats se penchera sur cet objet soumis par le DFI durant ce prochain mois d'avril.

Le plafond de coûts visé par l'article 47c ne ferait pas que limiter les prestations médicales et alourdir les tâches administratives supportées par notre système de santé, mais, comme le montre l'avis rendu par le professeur Ueli Kieser, expert en droit des assurances sociales, il serait contraire à la Constitution. En vertu de l'assurance de base, les assurés peuvent prétendre à des prestations définies à l'avance. Limiter a posteriori ces prestations contreviendrait au principe d'assurance.

Il reste à espérer que ces arguments seront entendus au sein des cercles politiques. Car sinon, nous risquerions de sentir avec quelle immédiateté les décisions en matière de politique de santé se répercutent sur la santé. D’elles aussi peut dépendre la possibilité d'avoir accès ou non à un traitement.

1 Heure des questions du 15 mars 2021; question 21.7261 Bregy et question 21.7288 Schneeberger. 